Review

\title{
Honokiol and Magnolol as Multifunctional Antioxidative Molecules for Dermatologic Disorders
}

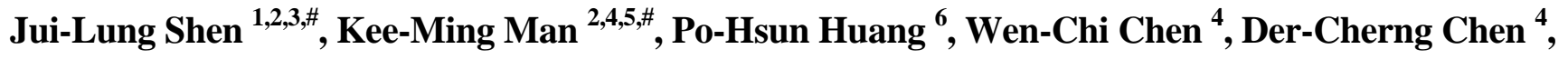 \\ Ya-Wen Cheng ${ }^{1}$, Po-Len Liu ${ }^{7, *}$, Ming-Chih Chou ${ }^{1, *}$, and Yung-Hsiang Chen ${ }^{4, *}$
}

1 Institute of Medicine, Chung Shan Medical University, Taichung; Taiwan; E-Mails: shen5300@yahoo.com.tw (J.L.S.); yw0727@mail2000.com.tw (Y.W.C.)

2 Department of Anesthesiology, Tungs' Taichung MetroHarbor Hospital, Taichung; Taiwan; E-Mail: man_jimmy60@hotmail.com (K.M.M.)

3 Department of Dermatology, Taichung Veterans General Hospital, Taichung; Taiwan

4 Graduate Institute of Integrated Medicine, College of Chinese Medicine, Graduate Institute of Clinical Medical Science, Department of Urology, Department of Neurosurgery, China Medical University and Hospital, Taichung; Taiwan; E-Mails: wgchen@mail.cmu.edu.tw (W.C.C.); vincenchen1966@gmail.com (D.C.C.); yhchen@mail.cmu.edu.tw (Y.H.C.)

5 Graduate Institute of Geriatric Medicine, Anhui Medical University, Hefei; China

6 Division of Cardiology, Taipei Veterans General Hospital, Institute of Clinical Medicine, Cardiovascular Research Center, National Yang-Ming University, Taipei; Taiwan; E-Mail: huangbsvgh@gmail.com

7 Department of Respiratory Therapy, College of Medicine, Kaohsiung Medical University, Kaohsiung; Taiwan; E-Mail: kisa@kmu.edu.tw

\# Jui-Lung Shen and Kee-Ming Man contributed equally to this study.

* Author to whom correspondence should be addressed; E-mail: kisa@kmu.edu.tw (P.L.); cs1601@csmu.edu.tw (M.C.); yhchen@mail.cmu.edu.tw (Y.C.); Tel.: +886-4-22053366-3512; Fax: +886-4-2203-7690.

Received: 27 August 2010; in revised form: 7 September 2010 / Accepted: 15 September 2010 / Published: 16 September 2010

\footnotetext{
Abstract: Chinese herbs have been and still are widely used as important remedies in Oriental medicine. Over the recent years, a variety of biologically active constituents have been isolated from these sources and confirmed to have multifunctional activity in experimental studies. Honokiol is a small-molecule polyphenol isolated from the genus Magnolia. It is accompanied by other related polyphenols, including magnolol, with which
} 
it shares certain biological properties. Recently, honokiol and magnolol have been found to have anti-oxidative, anti-inflammatory, anti-tumor, and anti-microbial properties in preclinical models, without appreciable toxicity. These findings have increased interest in bringing honokiol and magnolol to the clinic as novel therapeutic agents in dermatology. In this review, the findings concerning the major mechanisms of action of honokiol and magnolol are described. Knowledge of the multiple activities of honokiol and magnolol can assist with the development of honokiol and magnolol derivatives and the design of clinical trials that will maximize the potential benefit of honokiol and magnolol in the patient setting for dermatologic disorders.

Keywords: antibacterial; antioxidant; Chinese medicine; dermatology; honokiol; inflammation; magnolol

\section{Introduction}

Herbal therapy is becoming increasingly popular among physicians and patients [1,2]. Many medical plant preparations are marketed to the public for various ailments, including those of the skin [3-5]. Herbal therapies have been used successfully in Asia and Europe for treating dermatologic disorders (such as acne, wounds and burns, bacterial and fungal infections, dermatitis and psoriasis, and skin tumors) for thousands of years. In Asia, herbal treatments that have been used for centuries are now being studied scientifically. In United States, the herbal products are considered as dietary supplements or cosmetic additives. Since there is no standardization of active ingredients, purity, or concentration, this has made learning about and using these treatments challenging [6].

Figure 1. The chemical structures of (A) honokiol and (B) magnolol.

A

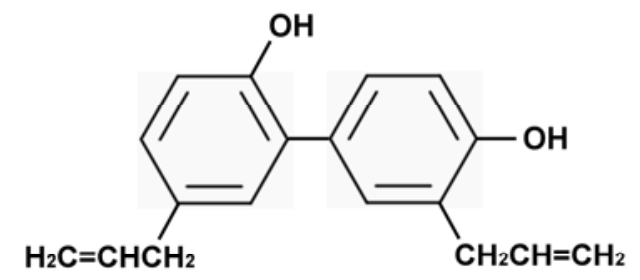

B

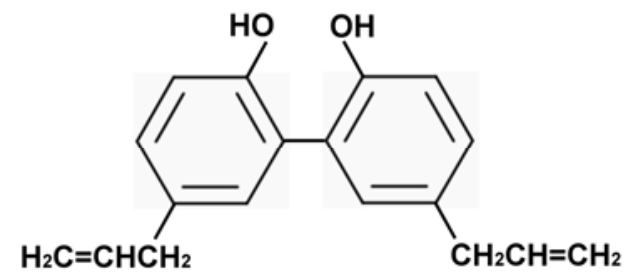

Honokiol and magnolol (Figure 1) were initially described as components of the genus Magnolia, which are components of Chinese (Kampo) herbs, including houpo and saiboku-tu(o) [7]. In the 1990s, 
honokiol and magnolol were found to have activity as free radical and lipid peroxidation inhibitors [8-11]. Thereafter, honokiol, magnolol, and a methanolic extract of Magnolia were shown to exhibit antioxidative, anti-inflammatory, anti-tumor, anti-diabetic complications, anti-microbial, anti-neurodegeneration, anti-depressant, pain control, hormone, gastrointestinal, and uterus modulation, cardiovascular and liver protective properties (Figure 2). The focus of this review are the recent findings regarding the biological effects of the antioxidative molecules honokiol and magnolol in dermatology (Table 1). Knowledge of the multiple biological activities of honokiol and magnolol could assist with the development of honokiol and magnolol derivatives and the design of clinical trials that will maximize the potential benefit of honokiol and magnolol in the patient setting for dermatologic disorders $[12,13]$.

Figure 2. The flower, leaf, and bark of traditional Chinese medicinal plant Magnolia officinalis and the medicinal properties of honokiol/magnolol.

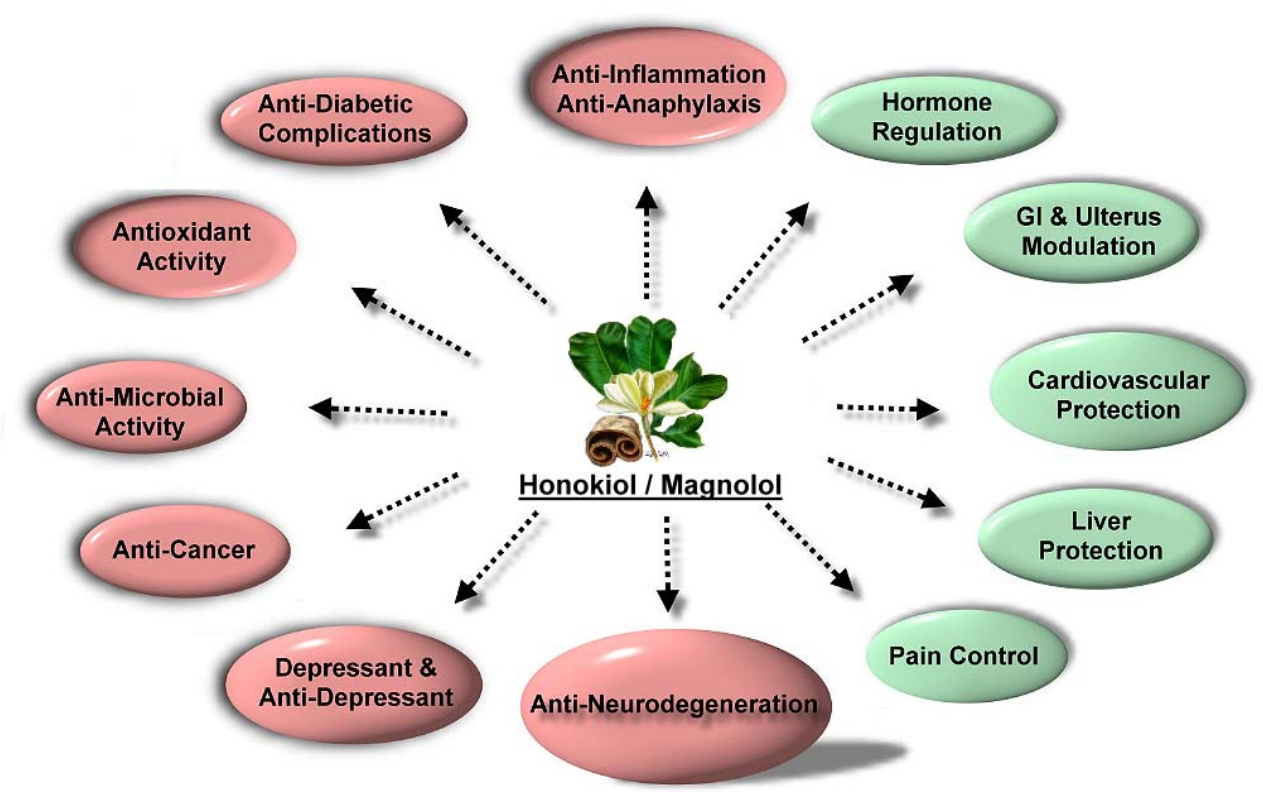

\section{General Antioxidative Effects}

This inhibition of oxidative stress was demonstrated in vitro; honokiol and magnolol reduce free radicals that generated by ultra-violet (UV) irradiation and inhibit UV-induced mutation in Salmonella [10]. This inhibition of lipid peroxidation was then ex vivo demonstrated in rat heart/liver mitochondria and human sperm [11,14]. It is approximately 1,000 times more potent than $\alpha$-tocopherol $[11,15]$ in inhibiting lipid peroxidation in heart mitochondria and 340 times more potent in rat liver mitochondria [14]. In the in vivo animal studies, magnolol protects against small intestinal, cerebral, and hind limb ischemia-reperfusion injury [16-19].

The in vitro cell culture system reveals the possible underlying mechanisms that honokiol and/or magnolol inhibit xanthine oxidase [20], protects mitochondrial respiratory chain enzyme, attenuates extracellular signal-regulated kinase (ERK) activation, and suppresses protein kinase $\mathrm{C}$ (PKC) and NADPH oxidase activities, resulting in the neutrophil respiratory burst inhibition [21] and cellular protection [17,22]. Dikalov et al. studied the reactions of honokiol in cell-free and cellular systems 
using electron spin resonance (ESR) and high-performance liquid chromatography (HPLC) techniques suggesting that honokiol is an effective scavenger of free radicals [23]. Additionally, the polyphenols may prevent formation of hydroxyl radical by chelating the transition metals such as copper and iron or repair molecules after free radical attack.

Table 1. Anti-oxidative, anti-inflammatory, anti-tumor, and anti-microbial effects and related mechanisms/outcomes of honokiol and/or magnolol.

\begin{tabular}{|c|c|c|}
\hline Biological Effect & Mechanism / Outcome & Reference \\
\hline \multicolumn{3}{|l|}{ I. General Antioxidant Activity } \\
\hline $\begin{array}{l}\text { Protection of heart mitochondria against } \\
\text { lipid peroxidation }\end{array}$ & Free radical scavenging activity & {$[11]$} \\
\hline Inhibition of xanthine oxidase & Antioxidative function & [20] \\
\hline $\begin{array}{l}\text { Inhibition of UV-induced mutation in } S \text {. } \\
\text { typhimurium }\end{array}$ & $\begin{array}{l}\text { Scavenger of free radicals generated by UV } \\
\text { irradiation }\end{array}$ & {$[10]$} \\
\hline Protection of sperm motility & Inhibition of lipid peroxidation & [73] \\
\hline $\begin{array}{l}\text { Protection of biological systems and } \\
\text { functions }\end{array}$ & $\begin{array}{l}\text { Protection of red cells and mitochondrial } \\
\text { respiratory chain enzyme activity; against } \\
\text { NADPH-induced peroxidative stress }\end{array}$ & {$[22]$} \\
\hline $\begin{array}{l}\text { Inhibition of fMLP-induced respiratory } \\
\text { burst in neutrophils }\end{array}$ & $\begin{array}{l}\text { Attenuation of ERK activation, and } \\
\text { suppression of PKC and NADPH oxidase } \\
\text { activities }\end{array}$ & {$[21]$} \\
\hline $\begin{array}{l}\text { Protective effect on the small intestinal } \\
\text { I/R injury }\end{array}$ & Antioxidative function & {$[18,19]$} \\
\hline Against heatstroke reactions & $\begin{array}{l}\text { Against cerebral ischemic injury by } \\
\text { antioxidative activity }\end{array}$ & {$[16]$} \\
\hline $\begin{array}{l}\text { Against cell killing, DNA damage, and lipid } \\
\text { peroxidation }\end{array}$ & Antioxidative function & {$[17]$} \\
\hline $\begin{array}{l}\text { Protective efficacy in hind limb ischemia- } \\
\text { reperfusion injury }\end{array}$ & $\begin{array}{l}\text { Antioxidant, anti-nitrosative, and anti- } \\
\text { inflammatory actions }\end{array}$ & {$[74]$} \\
\hline Effective scavenger of ROS & Potent scavenger of free radicals & {$[23]$} \\
\hline
\end{tabular}

\section{Anti-Inflammation and Anti-Tumor}

Inhibitory effects on mouse skin tumor promotion

Anti-inflammatory and analgesic effects

Inhibition of plasma leakage in passive cutaneous anaphylactic reaction, neurogenic inflammation, dorsal skin and ear edema
Inhibition of EBV-EA activation induced by TPA

Decrease of myeloperoxidase activity

Nonselective inhibition on vascular tissue to prevent the permeability change caused by various mediators 
Table 1. Cont.

Inhibition of A23187-induced pleurisy

Inhibition of the reduction of phorbol esterinduced neutrophil aggregation

Inhibition macrophage activation

Clinical efficacy in patients with asthma

Inhibition NO production in LPS-activated macrophages

Against allergy and anaphylaxis

Decrease in the excitability of airway myocytes

Inhibition of smooth muscle contraction in trachea

Influence of eicosanoid metabolism in neutrophils

Early protection against endotoxin challenge (following sub-lethal hemorrhage)

Anti-inflammatory effect of on neutrophils Inhibitory effect on tumor metastasis

Prevention of skin photoaging

Against passive cutaneous anaphylaxis reaction and scratching behaviors

Inhibition of various inflammatory events mediated by monocytes/macrophages and lymphocytes

Suppression of NF- $\kappa \mathrm{B}$ activation and NF$\kappa \mathrm{B}$ regulated gene expression

Inhibition of proliferation of malignant melanoma cells

Chemopreventive effects on UVB-induced skin cancer development
Reduction of eicosanoids mediator formation in the inflammatory site; suppression of PAF production in PMNs

Suppression of PKC activity; induction of cytosolic-free $\mathrm{Ca}^{2+}$ elevated neutrophils via IP3 signaling pathway

Inhibition of NO and TNF- $\alpha$ production in LPS-activated macrophages

Suppression of LTC4 release; inhibition of leukocyte leukotriene release; inhibition of $11 \beta$-hydroxysteroid dehydrogenase

Inhibition of NF- $\kappa \mathrm{B}$ activation

Anti-histamine release on mast cells; inhibition of PLA2, 5-LO, LTC4 synthase and LTA4 hydrolase which are essential for LT-synthesis

Stimulation of $\mathrm{BK}_{\mathrm{Ca}}$ channel activity in tracheal smooth muscle cells

Blockade of $\mathrm{Ca}^{2+}$ influx through voltageoperated $\mathrm{Ca}^{2+}$ channels instead of $\mathrm{Ca}^{2+}$ release from intracellular $\mathrm{Ca}^{2+}$ stores

Inhibition of prostaglandin and leukotriene formation

Alteration of the course of endotoxin tolerance and cytokine response; attenuation of peroxidative damage

Inhibition of ROS production

Ability to inhibit tumor cell invasion

Inhibition of bFGF and MMP-1

Inhibition of IL-4 and TNF- $\alpha$

Suppression of PI3K/Akt pathway

Inhibition of $\mathrm{I} \kappa \mathrm{B}$ kinase activation

Activation of both mitochondrial and death receptor pathways

Activating pro-apoptotic proteins through both intrinsic and extrinsic pathways 
Table 1. Cont.

\section{Anti-Microbial Activity}

Antifungal Activity

Acne-mitigating Activity

Antibacterial Activity

Synthesis and microbiological evaluation of honokiol derivatives as new antimicrobial agents
Against T. mentagrophytes, M. gypseum, E. floccosum, A. niger, C. neoformans, and $C$. albicans

Against Propionibacterium sp. and reduces secretion of IL- 8 and TNF- $\alpha$ induced by $P$. acnes in THP-1 cells; inhibition of downstream $\quad[59,60]$

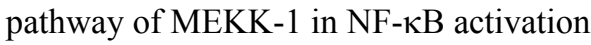
signaling

Bactericidal against VRE and MRSA; Against A. actinomycetemcomitans, $P$. gingivalis, $P$. intermedia, M. luteus, and B. subtilis; anti- $H$. pylori activity

Honokiol-glycine showed improved water solubility and antibacterial activities against $E$. coli and $P$. aeruginosa
$[56,57]$

\section{Anti-Inflammation and Anti-Tumor Effects}

Oxidative stress and inflammation [24] play important roles in skin tumor promotion [25]. Skin cancer is the most prevalent of all cancer types and its incidence is expected to increase substantially [26]. Chemoprevention involves the administration of chemical agents to prevent initiation, promotion and/or progression that occurs during neoplastic development [26,27]. Konoshima et al. were the first to test the Magnolia officinalis neolignans, honokiol and magnolol, for inhibition of Epstein-Barr virus early antigen (EBV-EA) activation induced by 12-O-tetradecanoylphorbol-13-acetate (TPA) and concluded these plant derivatives exhibited remarkable inhibitory effects on mouse skin tumor promotion in an in vivo carcinogenesis test [28]. Previous data showing blockade of inflammatory enzyme/cytokine production, nuclear factor (NF)- $\kappa$ B activation, and leukocyte activation suggest that honokiol and magnolol would have anti-inflammatory properties at clinically achievable concentrations [13].

Liou et al. found that honokiol inhibited PMA- or fMLP-induced reactive oxygen species (ROS) production by neutrophils by distinct mechanisms including: (i) honokiol diminished the activity of assembled-NADPH oxidase, a major reactive oxygen species producing enzyme in neutrophils; (ii) two other important enzymes for reactive oxygen species generation in neutrophils, i.e., myeloperoxidase and cyclooxygenase, were also inhibited by honokiol; and (iii) honokiol enhanced glutathione (GSH) peroxidase activity [29].

Immunological functions of magnolol and honokiol are important because incidences of skin diseases are also connected to immunological abnormalities. Honokiol/magnolol exhibits leukocyte suppression, anti-inflammation, and analgesic effects via decreasing myeloperoxidase activity [30], eicosanoids mediator [31-33] and leukotriene [34-39] formation, histamine release [37,40], as well as nitric oxide (NO), tumor necrosis factor- $\alpha(\mathrm{TNF}-\alpha)$, basic fibroblast growth factor (bFGF), matrix 
metalloproteinase (MMP)-1, and interleukin (IL)-4 production [41-43]. Magnolol inhibits passive cutaneous anaphylactic reaction [41], skin photoaging [43], neurogenic inflammation, as well as dorsal skin and ear edema [44]. The intracellular signaling pathways involved in the immunomodulation include the suppression of the PI3K/Akt pathway [45], PKC [46,47], and redox-sensitive transcription factor NF- $\kappa \mathrm{B}[48,49]$ activation, while the signal pathways and kinases upstream of $\mathrm{I} \kappa \mathrm{B}$ kinase (IKK) activation might be involved in the action of honokiol and magnolol [49].

Recent basic and clinical studies have implicated solar UV radiation in various skin diseases including premature aging and skin cancers. Chronic UV radiation exposure-induced skin disorders are caused by the excessive induction of inflammation, oxidative stress and DNA damage. The use of chemopreventive agents, such as plant polyphenols, to inhibit these events in UV-exposed skin is gaining attention [27]. More recently, in addition to chemically induced skin cancer development, Chilampalli et al. studied the chemopreventive effects of honokiol on UVB-induced skin tumor development in SKH-1 mice, a model relevant to humans, and to elucidate the possible role of apoptotic proteins involved in the prevention of skin tumor development. The honokiol-pretreated group exhibited significant reduction in tumor multiplicity as compared to the control group. Mechanistic studies showed the possible involvement of caspase-3, caspase-8, caspase-9, poly (ADP-ribose) polymerase (PARP) and p53 activation leading to the induction of DNA fragmentation and apoptosis. Their results show that honokiol acted as a potential chemopreventive agent to prevent UVB-induced skin cancer development, possibly by activating pro-apoptotic proteins through both intrinsic and extrinsic pathways [50]. These results suggested that honokiol and magnolol may favorably supplement sunscreen protection, and may be useful for skin diseases associated with solar UV radiation-induced inflammation, oxidative stress, and DNA damage.

Ikeda et al. investigated the anti-metastatic effect of magnolol on tumor metastasis in vivo with experimental and spontaneous metastasis models with an experimental and spontaneous lung metastasis model using melanoma to clarify the mechanism [51]. In addition, magnolol inhibited proliferation of human malignant melanoma cells. It induced oligonucleosomal fragmentation of DNA in melanoma cells and increased caspase-3, 8, 9 activities followed by the degradation of caspase-3 substrates, inhibitor of caspase dependent DNase (ICAD) and PARP indicating that magnolol induces apoptosis by activation of both mitochondrial and death receptor pathways in melanoma cells [52]. These data from the in vivo and in vitro experiments suggest that magnolol possesses strong anti-metastatic and tumor suppressing ability and that it may be a lead compound for drug development.

Additionally, magnolol provides early protection against endotoxin challenge (following sub-lethal hemorrhage) by altering the course of endotoxin tolerance and cytokine response and attenuating peroxidative damage [53-55]. These findings have increased interest in bringing magnolol to the clinic as a novel anti-inflammatory and anti-anaphylaxis agent for inflammation. Preparations containing magnolol have also been used in pilot clinical trials for inflammation-related disorders [34-36,38,39].

\section{Anti-Microbial Effects}

Clark et al. first tested the significant anti-microbial activity of magnolol using an agar well diffusion assay and found that honokiol and magnolol exhibited significant activity against Gram-positive and acid-fast bacteria and fungi [56,57]. The extract of Magnolia officinalis has been found to potently 
inhibit the growth of Helicobacter pylori [58]. Propionibacterium acnes, an anaerobic pathogen, plays an important role in the pathogenesis of acne and seems to initiate the inflammatory process by producing proinflammatory cytokines. Since magnolol had been known to exhibit antibacterial activities, Park et al. tested its antibacterial activity against Propionibacterium sp. In addition, they found the reduced secretion of IL- 8 and TNF- $\alpha$ induced by P. acnes in THP-1 cells indicating the anti-inflammatory effects of them [59]. Together with the previously known antibacterial activity against $P$. acnes and based on these results, it is suggested that magnolol may be introduced as possible acne-mitigating agents [60].

More recently, to improve the solubility and antibacterial activity of honokiol against $E$. coli and P. aeruginosa, new honokiol-derivatives (honokiol acetate, honokiol succinic acid, honokiol glycerol, honokiol glycine, honokiol glucose and honokiol mannose) were synthesized and their solubility and antimicrobial activities were investigated by Kim et al. They reported that among the tested compounds, honokiol glycine showed improved water solubility and antibacterial activities against E. coli and P. aeruginosa when compared to honokiol [61].

\section{Safety of Herbal Preparations}

There are many herbal therapies available for dermatological diseases that patients have already begun to discover $[62,63]$. Many patients have the misconception that these have no adverse effects because herbs are "natural" [64]. However, herbal preparations vary greatly in their therapeutic indexes [65]. For example, some are consumed as foods and have high therapeutic indexes, and others are highly biologically active and must be used very carefully [66]. Dermatologists must be educated not only in the benefits of these therapies, but must also be aware of some of the risks and potential adverse effects [67]. They need information about the effects of herbal remedies in order to better serve their patients who may be using herbs to treat their dermatological conditions [6]. In addition to the adverse effects, patients should be counseled on the lack of regulation for herbal medicines. The design of clinical trials should be encouraged to maximize the potential benefit of honokiol and magnolol in the patient setting for dermatologic disorders. Furthermore, quality-control measures to ensure the purity $[68,69]$, concentration [70], or safety of herbal supplements should be standardized scientifically based on evidence-base medicine data [71,72].

\section{Conclusions}

Honokiol and magnolol have shown strong antioxidative, anti-inflammatory, anti-tumor, and anti-microbial properties mediated by several modes of action. Thus, honokiol and magnolol exhibit a desirable spectrum of bioavailability rather than other natural products. To fully realize the potential of honokiol and magnolol, clinical trials are needed. Honokiol and magnolol analogues with improved pharmacokinetic and pharmacodynamics will also make the field move forward. Safety during long-term administration, combined with its cost and future therapeutic potential, makes it an ideal agent for both prevention and therapy in dermatology either alone or in combination with other drugs. This knowledge is required for the development of future analogues, which may target either of these pathways, and for the development of clinical trials using honokiol and magnolol or their analogues. Further insights into the signaling network and interaction points modulated by honokiol and magnolol 
may provide the basis for novel discovery programs to exploit honokiol and magnolol for the prevention and treatment of dermatologic disorders.

Figure 3. The protective effects of honokiol/magnolol for dermatologic disorders.

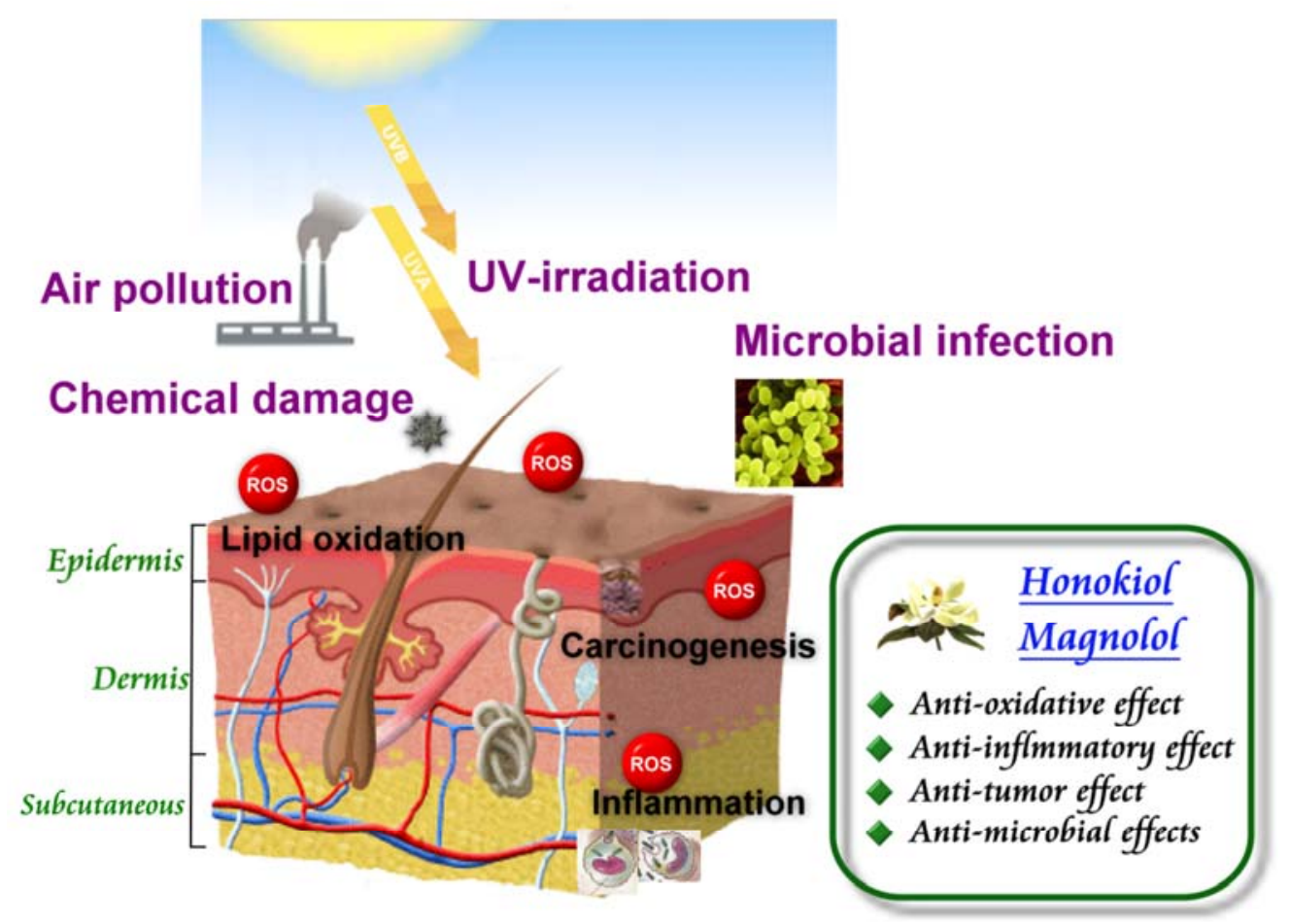

\section{Acknowledgements}

This study was supported by grants NSC 97-2320-B-039-022-MY3, NSC 97-2320-B-037-022-MY3, and NSC 98-2314-B-039-023-MY3 from the National Science Council, Taiwan and grant CMU99NTU-09 from China Medical University, Taichung, Taiwan. The authors thank Miss Jin-Mei Wang and Chi-Hsiang Wei for manuscript preparation.

\section{References}

1. Buchness, M. R. Alternative medicine and dermatology. Semin. Cutan. Med. Surg. 1998, 17 (4), 284-290.

2. Neldner, K. H. Complementary and alternative medicine. Dermatol. Clin. 2000, 18 (1), 189-193, xi.

3. Koo, J.; Desai, R. Traditional Chinese medicine in dermatology. Dermatol. Ther. 2003, 16 (2), 98-105.

4. Dattner, A. M. Herbal and complementary medicine in dermatology. Dermatol. Clin. 2004, 22 (3), 325-332, vii.

5. Baumann, L. Botanical ingredients in cosmeceuticals. J. Drugs Dermatol. 2007, 6 (11), 1084-1088.

6. Bedi, M. K.; Shenefelt, P. D. Herbal therapy in dermatology. Arch. Dermatol. 2002, 138 (2), 232-242.

7. Fujita, M.; Itokawa, H.; Sashida, Y. Studies on the components of Magnolia obovata Thunb. 3. Occurrence of magnolol and honokiol in M. obovata and other allied plants. Yakugaku Zasshi 1973, 93 (4), 429-434. 
8. Watanabe, H.; Watanabe, K.; Hagino, K. Chemostructural requirement for centrally acting muscle relaxant effect of magnolol and honokiol, neolignane derivatives. J. Pharmacobiodyn. 1983, 6 (3), 184-190.

9. Taira, J.; Ikemoto, T.; Mimura, K.; Hagi, A.; Murakami, A.; Makino, K. Effective inhibition of hydroxyl radicals by hydroxylated biphenyl compounds. Free Radic. Res. Commun. 1993, 19 (Suppl. 1), S71-S77.

10. Fujita, S.; Taira, J. Biphenyl compounds are hydroxyl radical scavengers: their effective inhibition for UV-induced mutation in Salmonella typhimurium TA102. Free Radic. Biol. Med. 1994, 17 (3), 273-277.

11. Lo, Y. C.; Teng, C. M.; Chen, C. F.; Chen, C. C.; Hong, C. Y. Magnolol and honokiol isolated from Magnolia officinalis protect rat heart mitochondria against lipid peroxidation. Biochem. Pharmacol. 1994, 47 (3), 549-553.

12. Hon, K. L.; Leung, T. F.; Ng, P. C.; Lam, M. C.; Kam, W. Y.; Wong, K. Y.; Lee, K. C.; Sung, Y. T.; Cheng, K. F.; Fok, T. F.; Fung, K. P.; Leung, P. C. Efficacy and tolerability of a Chinese herbal medicine concoction for treatment of atopic dermatitis: a randomized, double-blind, placebocontrolled study. Br. J. Dermatol. 2007, 157 (2), 357-363.

13. Fried, L. E.; Arbiser, J. L. A multifunctional antiangiogenic and antitumor agent. Antioxid Redox Signal 2009, 11 (5), 1139-1148.

14. Chiu, J. H.; Wang, J. C.; Lui, W. Y.; Wu, C. W.; Hong, C. Y. Effect of magnolol on in vitro mitochondrial lipid peroxidation and isolated cold-preserved warm-reperfused rat livers. J. Surg. Res. 1999, 82 (1), 11-16.

15. Hong, C. Y.; Huang, S. S.; Tsai, S. K. Magnolol reduces infarct size and suppresses ventricular arrhythmia in rats subjected to coronary ligation. Clin. Exp. Pharmacol. Physiol. 1996, 23 (8), 660-664.

16. Chang, C. P.; Hsu, Y. C.; Lin, M. T. Magnolol protects against cerebral ischaemic injury of rat heatstroke. Clin. Exp. Pharmacol. Physiol. 2003, 30 (5-6), 387-392.

17. Li, H. B.; Gao, J. M.; Ying, X. X.; Wang, S. P.; Li, J. C. Protective effect of magnolol on TBHPinduced injury in H460 cells partially via a p53 dependent mechanism. Arch. Pharm. Res. 2007, 30 (7), 850-857.

18. Loong, C. C.; Chiu, J. H.; Tiao, R. C.; Chiu, Y. Y.; Wu, C. W.; Lui, W. Y. Pretreatment with magnolol attenuates ischemia-reperfusion injury in rat small intestine. Transplant. Proc. 2001, 33 (7-8), 3737-3738.

19. Loong, C. C.; Chiu, J. H.; Tiao, R. C.; Chiu, Y. Y.; Wu, C. W.; Lui, W. Y. Protective effect of magnolol on the small intestinal ischemia-reperfusion injury. Transplant. Proc. 2002, 34 (7), 2679-2680.

20. Chang, W. S.; Chang, Y. H.; Lu, F. J.; Chiang, H. C. Inhibitory effects of phenolics on xanthine oxidase. Anticancer Res. 1994, 14 (2A), 501-506.

21. Wang, J. P.; Hsu, M. F.; Raung, S. L.; Chang, L. C.; Tsao, L. T.; Lin, P. L.; Chen, C. C. Inhibition by magnolol of formylmethionyl-leucyl-phenyl alanine-induced respiratory burst in rat neutrophils. J. Pharm. Pharmacol. 1999, 51 (3), 285-294.

22. Haraguchi, H.; Ishikawa, H.; Shirataki, N.; Fukuda, A. Antiperoxidative activity of neolignans from Magnolia obovata. J. Pharm. Pharmacol. 1997, 49 (2), 209-212. 
23. Dikalov, S.; Losik, T.; Arbiser, J. L. Honokiol is a potent scavenger of superoxide and peroxyl radicals. Biochem. Pharmacol. 2008, 76 (5), 589-596.

24. Graf, J. Herbal anti-inflammatory agents for skin disease. Skin Therapy Lett. 2000, 5 (4), 3-5.

25. Katiyar, S. K. Skin photoprotection by green tea: antioxidant and immunomodulatory effects. Curr. Drug Targets Immune Endocr Metabol Disord 2003, 3 (3), 234-242.

26. Halliday, G. M. Inflammation, gene mutation and photoimmunosuppression in response to UVRinduced oxidative damage contributes to photocarcinogenesis. Mutat. Res. 2005, 571 (1-2), 107-120.

27. Nichols, J. A.; Katiyar, S. K. Skin photoprotection by natural polyphenols: anti-inflammatory, antioxidant and DNA repair mechanisms. Arch. Dermatol. Res. 2010, 302 (2), 71-83.

28. Konoshima, T.; Kozuka, M.; Tokuda, H.; Nishino, H.; Iwashima, A.; Haruna, M.; Ito, K.; Tanabe, M. Studies on inhibitors of skin tumor promotion, IX. Neolignans from Magnolia officinalis. J. Nat. Prod. 1991, 54 (3), 816-822.

29. Liou, K. T.; Shen, Y. C.; Chen, C. F.; Tsao, C. M.; Tsai, S. K. The anti-inflammatory effect of honokiol on neutrophils: mechanisms in the inhibition of reactive oxygen species production. Eur J Pharmacol 2003, 475 (1-3), 19-27.

30. Wang, J. P.; Hsu, M. F.; Raung, S. L.; Chen, C. C.; Kuo, J. S.; Teng, C. M. Anti-inflammatory and analgesic effects of magnolol. Naunyn Schmiedebergs Arch. Pharmacol. 1992, 346 (6), 707-712.

31. Hsu, M. F.; Lu, M. C.; Tsao, L. T.; Kuan, Y. H.; Chen, C. C.; Wang, J. P. Mechanisms of the influence of magnolol on eicosanoid metabolism in neutrophils. Biochem. Pharmacol. 2004, 67 (5), 831-840.

32. Wang, J. P.; Ho, T. F.; Chang, L. C.; Chen, C. C. Anti-inflammatory effect of magnolol, isolated from Magnolia officinalis, on A23187-induced pleurisy in mice. J. Pharm. Pharmacol. 1995, 47 (10), 857-860.

33. Yamazaki, R.; Sugatani, J.; Fujii, I.; Kuroyanagi, M.; Umehara, K.; Ueno, A.; Suzuki, Y.; Miwa, M. Development of a novel method for determination of acetyl-CoA:1-alkyl-sn-glycero-3phosphocholine acetyltransferase activity and its application to screening for acetyltransferase inhibitors. Inhibition by magnolol and honokiol from Magnoliae cortex. Biochem. Pharmacol. 1994, 47 (6), 995-1006.

34. Homma, M.; Minami, M.; Taniguchi, C.; Oka, K.; Morita, S.; Niitsuma, T.; Hayashi, T. Inhibitory effects of lignans and flavonoids in saiboku-to, a herbal medicine for bronchial asthma, on the release of leukotrienes from human polymorphonuclear leukocytes. Planta Med. 2000, 66 (1), 88-91.

35. Homma, M.; Oka, K.; Niitsuma, T.; Itoh, H. A novel 11 beta-hydroxysteroid dehydrogenase inhibitor contained in saiboku-to, a herbal remedy for steroid-dependent bronchial asthma. J. Pharm. Pharmacol. 1994, 46 (4), 305-309.

36. Homma, M.; Oka, K.; Yamada, T.; Niitsuma, T.; Ihto, H.; Takahashi, N. A strategy for discovering biologically active compounds with high probability in traditional Chinese herb remedies: an application of saiboku-to in bronchial asthma. Anal. Biochem. 1992, 202 (1), 179-187.

37. Ikarashi, Y.; Yuzurihara, M.; Sakakibara, I.; Nakai, Y.; Hattori, N.; Maruyama, Y. Effects of the extract of the bark of Magnolia obovata and its biphenolic constituents magnolol and honokiol on histamine release from peritoneal mast cells in rats. Planta Med. 2001, 67 (8), 709-713. 
38. Niitsuma, T.; Morita, S.; Hayashi, T.; Homma, M.; Oka, K. Effects of absorbed components of saiboku-to on the release of leukotrienes from polymorphonuclear leukocytes of patients with bronchial asthma. Methods Find Exp. Clin. Pharmacol. 2001, 23 (2), 99-104.

39. Taniguchi, C.; Homma, M.; Takano, O.; Hirano, T.; Oka, K.; Aoyagi, Y.; Niitsuma, T.; Hayashi, T. Pharmacological effects of urinary products obtained after treatment with saiboku-to, a herbal medicine for bronchial asthma, on type IV allergic reaction. Planta Med. 2000, 66 (7), 607-611.

40. Hamasaki, Y.; Kobayashi, I.; Zaitu, M.; Tsuji, K.; Kita, M.; Hayasaki, R.; Muro, E.; Yamamoto, S.; Matsuo, M.; Ichimaru, T.; Miyazaki, S. Magnolol inhibits leukotriene synthesis in rat basophilic leukemia-2H3 cells. Planta Med. 1999, 65 (3), 222-226.

41. Han, S. J.; Bae, E. A.; Trinh, H. T.; Yang, J. H.; Youn, U. J.; Bae, K. H.; Kim, D. H. Magnolol and honokiol: inhibitors against mouse passive cutaneous anaphylaxis reaction and scratching behaviors. Biol. Pharm. Bull 2007, 30 (11), 2201-2203.

42. Son, H. J.; Lee, H. J.; Yun-Choi, H. S.; Ryu, J. H. Inhibitors of nitric oxide synthesis and TNF-alpha expression from Magnolia obovata in activated macrophages. Planta Med. 2000, 66 (5), 469-471.

43. Tanaka, K.; Hasegawa, J.; Asamitsu, K.; Okamoto, T. Magnolia ovovata extract and its active component magnolol prevent skin photoaging via inhibition of nuclear factor kappaB. Eur. J. Pharmacol. 2007, 565 (1-3), 212-219.

44. Wang, J. P.; Raung, S. L.; Chen, C. C.; Kuo, J. S.; Teng, C. M. The inhibitory effect of magnolol on cutaneous permeability in mice is probably mediated by a nonselective vascular hyporeactivity to mediators. Naunyn Schmiedebergs Arch. Pharmacol. 1993, 348 (6), 663-669.

45. Kim, B. H.; Cho, J. Y. Anti-inflammatory effect of honokiol is mediated by PI3K/Akt pathway suppression. Acta Pharmacol. Sin. 2008, 29 (1), 113-122.

46. Wang, J. P.; Chen, C. C. Magnolol induces cytosolic-free $\mathrm{Ca}^{2+}$ elevation in rat neutrophils primarily via inositol trisphosphate signalling pathway. Eur. J. Pharmacol. 1998, 352 (2-3), 329-334.

47. Wang, J. P.; Lin, P. L.; Hsu, M. F.; Chen, C. C. Possible involvement of protein kinase c inhibition in the reduction of phorbol ester-induced neutrophil aggregation by magnolol in the rat. J. Pharm. Pharmacol. 1998, 50 (10), 1167-72.

48. Matsuda, H.; Kageura, T.; Oda, M.; Morikawa, T.; Sakamoto, Y.; Yoshikawa, M. Effects of constituents from the bark of Magnolia obovata on nitric oxide production in lipopolysaccharideactivated macrophages. Chem. Pharm. Bull 2001, 49 (6), 716-720.

49. Tse, A. K.; Wan, C. K.; Zhu, G. Y.; Shen, X. L.; Cheung, H. Y.; Yang, M.; Fong, W. F. Magnolol suppresses NF-kappaB activation and NF-kappaB regulated gene expression through inhibition of IkappaB kinase activation. Mol. Immunol. 2007, 44 (10), 2647-2658.

50. Chilampalli, S.; Zhang, X.; Fahmy, H.; Kaushik, R. S.; Zeman, D.; Hildreth, M. B.; Dwivedi, C. Chemopreventive effects of honokiol on UVB-induced skin cancer development. Anticancer Res. 2010, 30 (3), 777-783.

51. Ikeda, K.; Sakai, Y.; Nagase, H. Inhibitory effect of magnolol on tumour metastasis in mice. Phytother. Res. 2003, 17 (8), 933-937.

52. You, Q.; Li, M.; Jiao, G. Magnolol induces apoptosis via activation of both mitochondrial and death receptor pathways in A375-S2 cells. Arch. Pharm. Res. 2009, 32 (12), 1789-1794.

53. Kong, C. W.; Tsai, K.; Chin, J. H.; Chan, W. L.; Hong, C. Y. Magnolol attenuates peroxidative damage and improves survival of rats with sepsis. Shock 2000, 13 (1), 24-28. 
54. Shih, H. C.; Wei, Y. H.; Lee, C. H. Magnolol alters cytokine response after hemorrhagic shock and increases survival in subsequent intraabdominal sepsis in rats. Shock 2003, 20 (3), 264-268.

55. Shih, H. C.; Wei, Y. H.; Lee, C. H. Magnolol alters the course of endotoxin tolerance and provides early protection against endotoxin challenge following sublethal hemorrhage in rats. Shock 2004, 22 (4), 358-363.

56. Bang, K. H.; Kim, Y. K.; Min, B. S.; Na, M. K.; Rhee, Y. H.; Lee, J. P.; Bae, K. H. Antifungal activity of magnolol and honokiol. Arch. Pharm. Res. 2000, 23 (1), 46-49.

57. Clark, A. M.; El-Feraly, F. S.; Li, W. S. Antimicrobial activity of phenolic constituents of Magnolia grandiflora L. J. Pharm. Sci. 1981, 70 (8), 951-952.

58. Bae, E. A.; Han, M. J.; Kim, N. J.; Kim, D. H. Anti-Helicobacter pylori activity of herbal medicines. Biol. Pharm. Bull 1998, 21 (9), 990-992.

59. Park, J.; Lee, J.; Jung, E.; Park, Y.; Kim, K.; Park, B.; Jung, K.; Park, E.; Kim, J.; Park, D. In vitro antibacterial and anti-inflammatory effects of honokiol and magnolol against Propionibacterium sp. Eur. J. Pharmacol. 2004, 496 (1-3), 189-195.

60. Lee, J.; Jung, E.; Park, J.; Jung, K.; Lee, S.; Hong, S.; Park, E.; Kim, J.; Park, S.; Park, D. Anti-inflammatory effects of magnolol and honokiol are mediated through inhibition of the downstream pathway of MEKK-1 in NF-kappaB activation signaling. Planta Med. 2005, 71 (4), 338-343.

61. Kim, Y. S.; Lee, J. Y.; Park, J.; Hwang, W.; Lee, J.; Park, D. Synthesis and microbiological evaluation of honokiol derivatives as new antimicrobial agents. Arch. Pharm. Res. 2010, 33 (1), 61-65.

62. Nicolaou, N.; Johnston, G. A. The use of complementary medicine by patients referred to a contact dermatitis clinic. Contact Dermatitis 2004, 51 (1), 30-33.

63. Zhong, S.; Wu, Y.; Soo-Mi, A.; Zhao, J.; Wang, K.; Yang, S.; Jae-Ho, Y.; Zhu, X. Depigmentation of melanocytes by the treatment of extracts from traditional Chinese herbs: a cell culture assay. Biol. Pharm. Bull 2006, 29 (9), 1947-1951.

64. Walker, P. S.; Donovan, J. A. Herbal remedies: natural caveats. Int. J. Dermatol. 1999, 38 (10), 746-748.

65. Boneberger, S.; Rupec, R. A.; Ruzicka, T. Complementary therapy for atopic dermatitis and other allergic skin diseases: facts and controversies. Clin. Dermatol. 2010, 28 (1), 57-61.

66. Ernst, E. Adverse effects of herbal drugs in dermatology. Br. J. Dermatol. 2000, 143 (5), 923-929.

67. Dattner, A. M. From medical herbalism to phytotherapy in dermatology: back to the future. Dermatol. Ther. 2003, 16 (2), 106-113.

68. Keane, F. M.; Munn, S. E.; Vivier, A. W.; Higgins, E. M.; Taylor, N. F. Analysis of Chinese herbal creams prescribed for dermatological conditions. West J. Med. 1999, 170 (5), 257-259.

69. Keane, F. M.; Munn, S. E.; du Vivier, A. W.; Taylor, N. F.; Higgins, E. M. Analysis of Chinese herbal creams prescribed for dermatological conditions. BMJ 1999, 318 (7183), 563-564.

70. Wang, K. H.; Lin, R. D.; Hsu, F. L.; Huang, Y. H.; Chang, H. C.; Huang, C. Y.; Lee, M. H. Cosmetic applications of selected traditional Chinese herbal medicines. J. Ethnopharmacol. 2006, 106 (3), 353-359.

71. Hughes, R.; Ward, D.; Tobin, A. M.; Keegan, K.; Kirby, B. The use of alternative medicine in pediatric patients with atopic dermatitis. Pediatr. Dermatol. 2007, 24 (2), 118-120. 
72. Salameh, F.; Perla, D.; Solomon, M.; Gamus, D.; Barzilai, A.; Greenberger, S.; Trau, H. The effectiveness of combined Chinese herbal medicine and acupuncture in the treatment of atopic dermatitis. J. Altern. Complement. Med. 2008, 14 (8), 1043-1048.

73. Lin, M. H.; Chao, H. T.; Hong, C. Y. Magnolol protects human sperm motility against lipid peroxidation: a sperm head fixation method. Arch. Androl. 1995, 34 (3), 151-156.

74. Chen, H. Y.; Hung, Y. C.; Lee, E. J.; Chen, T. Y.; Chuang, I. C.; Wu, T. S. The protective efficacy of magnolol in hind limb ischemia-reperfusion injury. Phytomedicine 2009, 16 (10), 976-981.

75. Wu, S. N.; Chen, C. C.; Li, H. F.; Lo, Y. K.; Chen, S. A.; Chiang, H. T. Stimulation of the BK(Ca) channel in cultured smooth muscle cells of human trachea by magnolol. Thorax 2002, 57 (1), 67-74.

76. Ko, C. H.; Chen, H. H.; Lin, Y. R.; Chan, M. H. Inhibition of smooth muscle contraction by magnolol and honokiol in porcine trachea. Planta Med. 2003, 69 (6), 532-536.

77. Syu, W. J.; Shen, C. C.; Lu, J. J.; Lee, G. H.; Sun, C. M. Antimicrobial and cytotoxic activities of neolignans from Magnolia officinalis. Chem. Biodivers. 2004, 1 (3), 530-537.

78. Ho, K. Y.; Tsai, C. C.; Chen, C. P.; Huang, J. S.; Lin, C. C. Antimicrobial activity of honokiol and magnolol isolated from Magnolia officinalis. Phytother. Res. 2001, 15 (2), 139-141.

(C) 2010 by the authors; licensee MDPI, Basel, Switzerland. This article is an open access article distributed under the terms and conditions of the Creative Commons Attribution license (http://creativecommons.org/licenses/by/3.0/). 